\title{
Detection of buckminsterfullerene emission in the diffuse interstellar medium
}

\author{
O. Berné ${ }^{1,2}$, N. L. J. Cox ${ }^{1,2}$, G. Mulas ${ }^{3}$, and C. Joblin ${ }^{1,2}$ \\ 1 Université de Toulouse, UPS-OMP, IRAP, 31028 Toulouse, France \\ e-mail: olivier . berne@gmail.com \\ 2 CNRS, IRAP, 9 Av. colonel Roche, BP 44346, 31028 Toulouse Cedex 4, France \\ 3 Istituto Nazionale di Astrofisica, Osservatorio Astronomico di Cagliari, strada 54, localit Poggio dei Pini, 09012 Capoterra (CA), \\ Italy
}

Received 22 December 2016 / Accepted 20 June 2017

\begin{abstract}
Emission of fullerenes in their infrared vibrational bands has been detected in space near hot stars. The proposed attribution of the diffuse interstellar bands at 9577 and $9632 \AA$ to electronic transitions of the buckminsterfullerene cation (i.e. $\mathrm{C}_{60}^{+}$) was recently supported by new laboratory data, confirming the presence of this species in the diffuse interstellar medium (ISM). In this Letter, we present the detection, also in the diffuse ISM, of the 17.4 and $18.9 \mu \mathrm{m}$ emission bands commonly attributed to vibrational bands of neutral $\mathrm{C}_{60}$. According to classical models that compute the charge state of large molecules in space, $\mathrm{C}_{60}$ is expected to be mostly neutral in the diffuse ISM. This is in agreement with the abundances of diffuse $\mathrm{C}_{60}$ we derive here from observations.
\end{abstract}

Key words. infrared: ISM - ISM: molecules - ISM: lines and bands

\section{Introduction}

Fullerenes are cage-like macromolecules made of carbon. The most emblematic member of this family, $\mathrm{C}_{60}$, i.e. buckminsterfullerene, was serendipitously discovered by Kroto et al. (1985) during experiments aimed at simulating carbon chemistry in the atmospheres of evolved stars. Fullerenes have been extensively studied in the field of chemistry because of their unique properties and potential applications for nanotechnologies. Soon after the discovery of these macromolecules, it was recognized that fullerenes could be present in space and constitute a family of species relevant to astrochemistry (Kroto et al. 1985; Kroto \& Jura 1992). In particular, it was postulated that their electronic transitions could be at the origin of some of the numerous unidentified absorption bands observed towards reddened stars, the so-called diffuse interstellar bands (DIBs). Foing \& Ehrenfreund (1994) proposed that two DIBs at 9577 and $9632 \AA$ could be due to the $\mathrm{C}_{60}$ cation, $\mathrm{C}_{60}^{+}$, but this proposal could not be confirmed at that time because of the lack of gas-phase laboratory spectroscopy. Clear evidence for the presence of fullerenes $\left(\mathrm{C}_{60}, \mathrm{C}_{60}^{+}, \mathrm{C}_{70}\right)$ in space was provided recently with the detection of infrared (IR) emission bands of fullerenes in regions with intense UV radiation such as evolved stars (e.g. Cami et al. 2010; García-Hernández et al. 2010) and star-forming regions (Sellgren et al. 2010; Roberts et al. 2012; Berné et al. 2013; Castellanos et al. 2014). In parallel, new gas-phase laboratory experiments (Campbell et al. 2015) provided an accurate wavelength determination of the electronic transitions of $\mathrm{C}_{60}^{+}$, which were found to be in excellent agreement with observations, therefore supporting the hypothesis that the 9577 and $9632 \AA$ DIBs are due to the presence of $\mathrm{C}_{60}^{+}$in the diffuse interstellar medium (ISM). If fullerenes are present in the diffuse ISM, they should absorb ultraviolet (UV) photons and re-emit their energy in the IR, hence their IR vibrational bands should be observed. Unfortunately, in the diffuse ISM, the exciting UV radiation field is orders of magnitude smaller compared to star-forming regions, resulting in extremely weak IR fluorescence. For this reason fullerene emission has not been detected there so far, precluding an independent confirmation of their presence in this environment.

\section{Observations}

We retrieved archival IR spectra obtained with the InfraRed Spectrograph (IRS; Houck et al. 2004) on board Spitzer (Werner et al. 2004a) towards two lines of sight. To complement this data, we used the ISOCAM (Cesarsky et al. 1996) data, which was published in Flagey et al. (2006). An overview of the studied regions and data are presented in Fig. 1.

The first line of sight (Fig. 1, left column, Chamaeleon), corresponds to a translucent cloud that is situated on the frontside of the Chamaeleon complex at a distance of $\sim 70 \mathrm{pc}$ (Mizuno et al. 2001). As can be seen from the HI and CO spectra (Fig. 1), this is a single cloud. The physical conditions for this cloud have been derived by Ingalls et al. (2011) based on the study of the molecular hydrogen emission: the hydrogen density is $\sim 30-100$ particles $\mathrm{cm}^{-3}$ and the ultraviolet (UV) radiation field there is in the range of $G_{0}=0.7-3$ (with $G_{0}=1$ corresponding to $1.2 \times 10^{-7} \mathrm{~W} \mathrm{~m}^{-2} \mathrm{sr}^{-1}$, the interstellar standard radiation field calculated by Habing 1968). The data for this line of sight consists of low resolution spectroscopy $(\lambda / \Delta \lambda \sim 60-100)$ covering the 5-35 $\mu \mathrm{m}$ range and published in Ingalls et al. (2011) who focused on $\mathrm{H}_{2}$ emission. The second line of sight (Fig. 1, right column, Scutum) corresponds to a diffuse Galactic region, free of star formation, situated close to the Scutum. As can be seen in the HI and CO spectra presented in Fig. 1, this line of 
Chamaeleon
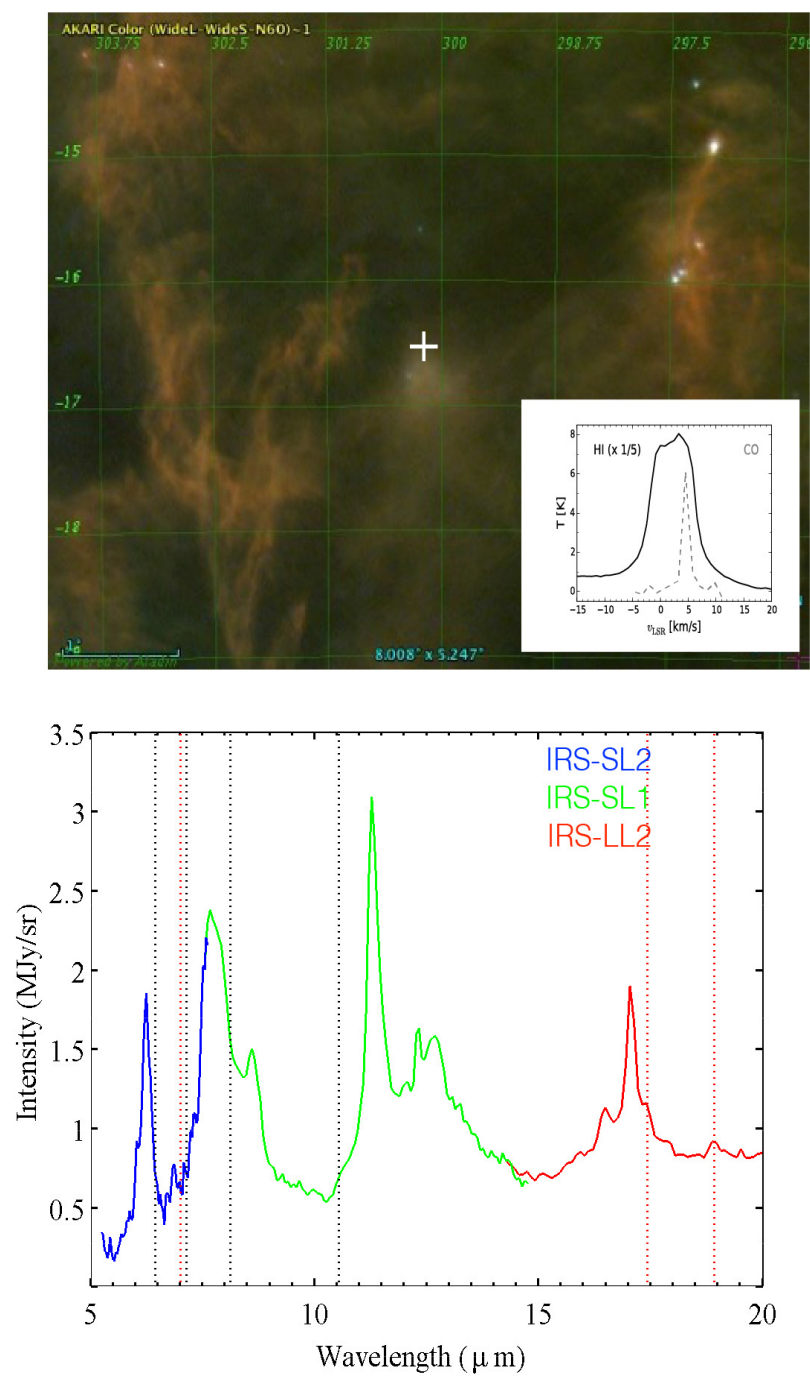

Scutum
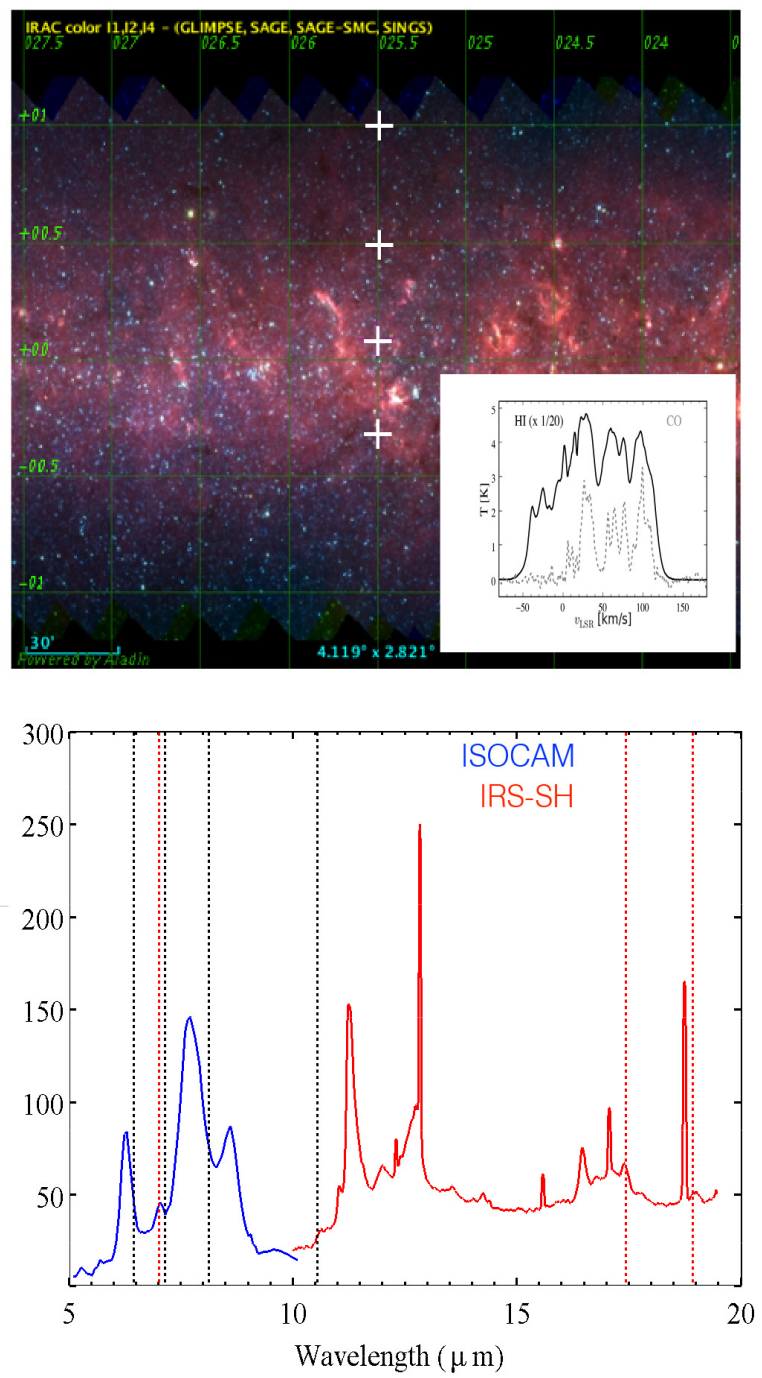

Fig. 1. Upper panels: images of the Chamaeleon (translucent) and Scutum (diffuse) lines of sight studied in this article, observed with SpitzerIRAC (Werner et al. 2004a; Fazio et al. 2004). Positions of the spectroscopic observations are indicated with white crosses. The images show IRAC colours red $=8.0 \mu \mathrm{m}$, green $=4.5 \mu \mathrm{m}$, and blue $=3.6 \mu \mathrm{m}$. Inserts show the $\mathrm{HI}$ and CO emission spectra (McClure-Griffiths et al. 2009; Dame et al. 2001; Boulanger et al. 1998). Lower panels: mid-infrared spectra towards the studied lines of sight are represented. The vertical lines indicate the positions of $\mathrm{C}_{60}$ (red) and $\mathrm{C}_{60}^{+}$(black) bands observed in the NGC 7023 reflection nebula (Sellgren et al. 2010; Berné et al. 2013). The band at $7.0 \mu \mathrm{m}$ observed in the diffuse line of sight is due to [ArII].

Table 1. Properties of the studied lines of sight.

\begin{tabular}{lccc}
\hline \hline & Chamaeleon & Scutum & Refs. \\
\hline Coord. $(G a l . /$ deg.) & $+300.13,-16.49$ & $26.47,-0.3-+1.0$ & $(1),(2)$ \\
UV field $G_{0}{ }^{*}$ & $0.7-3$ & $1-10$ & $(1),(3)$ \\
$N_{\mathrm{H}}\left(\mathrm{H} \mathrm{cm}^{-2}\right)$ & $2.7-5.6 \times 10^{21}$ & $5.7 \times 10^{22}$ & $(1),(3)$ \\
$A_{\mathrm{V}}{ }^{* *}(\mathrm{mag})$ & $1.4-3.0$ & 30.5 & - \\
Comment & Single translucent cloud & Multiple clouds & - \\
\hline
\end{tabular}

Notes. ${ }^{(*)} G_{0}=1$ corresponds to a UV field of $1.2 \times 10^{-7} \mathrm{~W} \mathrm{~m}^{-2} \mathrm{sr}^{-1}$. $^{(*)}$ Computed using $N_{\mathrm{H}} / A_{\mathrm{V}}=1.87 \times 10^{21}$ for Scutum (value for the diffuse ISM) and $N_{\mathrm{H}} / A_{\mathrm{V}}=3.1 \times 10^{21}$ for the Chamaelon (value for translucent/dense ISM).

References. (1): Ingalls et al. (2011); (2): this work; (3): Falgarone et al. (2005).

sight probes several clouds, where hydrogen can be in molecular, atomic, or ionized form. Falgarone et al. (2005) studied this region of the Galaxy in detail using ISO spectroscopy and were able to decompose the line of sight into several density components. The radiation field on this line of sight varies between
$G_{0}=1$ and $G_{0}=10$ (Falgarone et al. 2005). The Spitzer spectroscopic data for this line of sight was not previously published; it consists of medium resolution spectroscopy $(\lambda / \Delta \lambda \sim 600)$ covering the $9-20 \mu \mathrm{m}$ range. For both lines of sight, we reduced the data starting from the basic calibrated data and carefully 

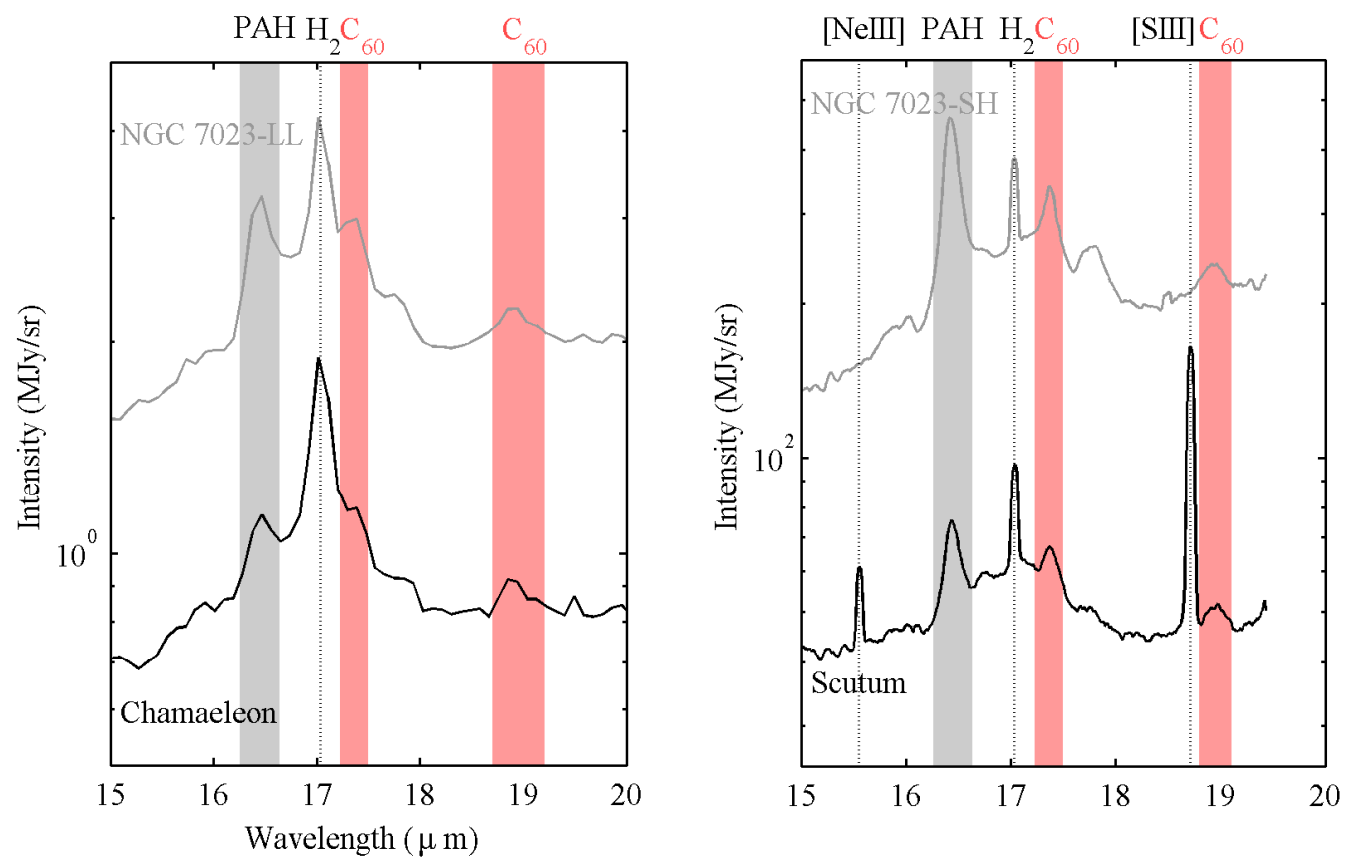

Fig. 2. Mid-infrared spectra extracted over the 15-20 $\mu \mathrm{m}$ range for both lines of sight (see Appendix A for details). The spectrum of the NGC 7023 nebula where $\mathrm{C}_{60}$ emission has been reported (Sellgren et al. 2010) is shown as a reference. The Chamaeleon spectrum is compared with a low resolution spectrum (IRS-LL) of NGC 7023, while the Scutum line of sight spectrum is compared to a medium resolution spectrum (IRS-SH) of NGC 7023, to be consistent with the observing modes used to obtain the spectra of interest here (see Appendix A). The positions of the $\mathrm{C}_{60}$ vibrational bands are indicated with red vertical patches. The $16.4 \mu \mathrm{m}$ attributed to PAH emission is indicated with a grey patch. Spectrally unresolved lines of molecular hydrogen and ions are also indicated with vertical dotted lines.

subtracting background emission (see Appendix A). The extracted mid-IR spectra over the full spectral range for the two lines of sight are presented in Fig. 1.

In Fig. 2, the same spectra are shown over a smaller spectral range that is of interest when searching for the emission of $\mathrm{C}_{60}$. For comparison, the spectra of the NGC 7023 massive star-forming region are shown, where $\mathrm{C}_{60}$ was detected by Sellgren et al. (2010) and where the UV radiation field is much higher, i.e. $G_{0}=1000-10000$. The diffuse and translucent cloud spectra show a number of broad features, which are commonly attributed to the emission of large carbonaceous molecules i.e. polycyclic aromatic hydrocarbons (PAH; Tielens 2008). The ratio between the 11.2 and $7.7 \mu \mathrm{m}$ PAH bands observed in both lines of sight is compatible with PAHs being mostly neutral (Pilleri et al. 2012). The molecular hydrogen lines at $17.0 \mu \mathrm{m}$ and $12.3 \mu \mathrm{m}$ are also observed towards both lines of sight. In addition, ionic lines from the warm ionized medium are detected towards the Scutum line of sight. The two most intense bands of $\mathrm{C}_{60}$, situated at 17.4 and $18.9 \mu \mathrm{m}$, are clearly detected in both lines of sight (Fig. 2) ${ }^{1}$. The vibrational spectrum of $\mathrm{C}_{60}$ is characterized by two additional weaker bands at 7.0 and $8.5 \mu \mathrm{m}$, which are not detected. However this non-detection is consistent with the noise level that exceeds the expected intensity for these bands. The $18.9 \mu \mathrm{m}$ band is detected at a level that is 3.2 and 12.2 times above the root mean square noise, for the Chameleon cloud and for the Scutum line of sight, respectively. The strongest bands of $\mathrm{C}_{60}^{+}$(at 6.4 and $7.1 \mu \mathrm{m}$, Berné et al. 2013) are not detected. $\mathrm{C}_{60}^{+}$also has an emission band close to $18.9 \mu \mathrm{m}$, which could correspond to the emission band detected the spectra in Fig. 2, but we show in Appendix F that $\mathrm{C}_{60}^{+}$cannot contribute more than $\sim 25 \%$ to the observed band.

\footnotetext{
1 The $17.4 \mu \mathrm{m}$ can be contaminated by emission from polycyclic aromatic hydrocarbons.
}

\section{Abundances of $\mathrm{C}_{60}$}

Using the integrated intensity of the $18.9 \mu \mathrm{m}$ band of $\mathrm{C}_{60}$ and information reported in Table 1 , it is possible to estimate an abundance for $\mathrm{C}_{60}$ in both lines of sight (see Appendix B). The values found are of the order of a few $10^{-4}$ to a few $10^{-3}$ of the gas-phase carbon locked in $\mathrm{C}_{60}$ (Table 2). This can be compared to the abundances of $\mathrm{C}_{60}^{+}$for the diffuse ISM derived from DIB measurements. Using the recent laboratory measurements for the oscillator strength of $\mathrm{C}_{60}^{+}$electronic transitions (Campbell et al. 2016), we derived the abundance of $\mathrm{C}_{60}^{+}$towards lines of sight where the $9577 \AA$ DIB has been detected (Appendix C). These abundances are in the range of $0.6-1.1 \times 10^{-3}$ of the carbon (Table C.1), i.e. comparable to what is found for $\mathrm{C}_{60}$ in this study; they are also in agreement with the upper limit for the abundance of $\mathrm{C}_{60}^{+}$we derived from the non-detection of the infrared bands of this species of $1.8 \times 10^{-3}$ of the carbon (see Appendix D). Abundances for $\mathrm{C}_{60}$ in the diffuse ISM, star-forming regions and evolved stars are summarized in Table 2. Fullerenes are most abundant in carbon-rich evolved stars, roughly an order of magnitude less abundant in the diffuse ISM, and two orders of magnitude less abundant in star forming regions. It should however be noted that $\mathrm{C}_{60}$ is detected towards only $\sim 3 \%$ of the evolved stars observed with Spitzer (Otsuka et al. 2014). Overall, the relatively large abundance of $\mathrm{C}_{60}$ in the diffuse ISM may therefore reflect the long-term processing of carbonaceous material by UV photons of massive stars, which can lead to the formation of fullerenes (Berné \& Tielens 2012; Berné et al. 2015).

According to classical theoretical models for the charge state of large molecules in space, $\mathrm{C}_{60}$ should be mostly neutral for standard physical conditions of the diffuse ISM. Bakes \& Tielens (1995) computed the charge distribution for the specific case of $\mathrm{C}_{60}$ in the diffuse ISM and find that $\sim 30 \%$ is 
Table 2. Abundances of fullerenes (\% of gas-phase carbon locked in species), derived from emission or absorption measurements in starforming regions and in the diffuse ISM and evolved stars.

\begin{tabular}{ccc}
\hline \hline & Emission & Absorption \\
\hline \multicolumn{4}{c}{ Star-forming regions } \\
\hline $\mathrm{C}_{60}^{+}$ & $0.01^{*}$ & - \\
$\mathrm{C}_{60}$ & $0.04-0.06^{* *}$ & - \\
\hline \multicolumn{4}{c}{ Diffuse ISM } \\
\hline $\mathrm{C}_{60}^{+}$ & $0.2^{\star}$ † $^{\star}$ & $0.06-0.1^{\star}$ \\
$\mathrm{C}_{60}$ & $0.03-0.4^{\star}$ & - \\
\hline \multicolumn{4}{c}{ Evolved stars } \\
\hline $\mathrm{C}_{60}^{+}$ & - & $1.2^{\star \star}$ \\
$\mathrm{C}_{60}$ & $0.1-3.0^{\star \star \star}$ & - \\
\hline
\end{tabular}

Notes. ${ }^{(*)}$ From Berné et al. (2013); ${ }^{(*)}$ from Castellanos et al. (2014);

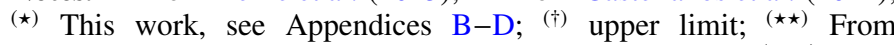
Iglesias-Groth \& Esposito (2013), for one source only; ${ }^{(\star \star \star)}$ from Cami et al. (2011) and references therein.

anionic, $\sim 60 \%$ is neutral, and $\sim 10 \%$ is cationic. These numbers are compatible with the absolute abundances summarized in Table 2, which point to a (not very restrictive) $\mathrm{C}_{60}$ over $\mathrm{C}_{60}^{+}$ratio ranging between 0.3 to 6 . $\mathrm{C}_{60}^{-}$is expected to be abundant in models and hence could be searched for in space. The strongest vibrational bands of $\mathrm{C}_{60}^{-}$are around 17.5 and $7.3 \mu \mathrm{m}$ (Kupser et al. 2008). The $17.5 \mu \mathrm{m}$ band is difficult to identify because of the presence of PAH emission at $17.4 \mu \mathrm{m}$. A band at $7.3 \mu \mathrm{m}$ seems present in the spectrum of the Chamaeleon line of sight, however this region of the spectrum is quite noisy and higher sensitivity data will be required to confirm this. Observations of diffuse lines of sight with the James Webb Space Telescope, which has the sensitivity to detect $\mathrm{C}_{60}$ and $\mathrm{C}_{60}^{+}$as well as tracers of the diffuse ISM should allow us to conduct more detailed studies on the charge balance of $\mathrm{C}_{60}$ in the diffuse ISM. Laboratory studies to quantify key molecular parameters involved, such as the electron recombination rate of $\mathrm{C}_{60}^{+}$, are also required.

Finally, the detection of $\mathrm{C}_{60}$ IR emission bands in the diffuse ISM suggests that the electronic transitions of this molecules could be identified in absorption. However, given their low oscillator strengths, this will be challenging as we illustrate in Appendix E.

\section{Conclusion}

In the past few years, our understanding of the organic inventory in space has greatly benefited from studies of the infrared emission of the Galaxy, in particular with the first identifications of fullerenes. Meanwhile, the field of diffuse interstellar bands has been reinvigorated by the convincing assignment of the 9577 and $9632 \AA$ DIBs to the electronic transitions of $\mathrm{C}_{60}^{+}$. The detection of $\mathrm{C}_{60}^{+}$IR emission in the diffuse ISM, with the same abundance as those derived from studies in absorption, would provide a strong independent confirmation of the DIB assignment. It is also likely that astronomical observations combining vibrational and electronic spectroscopy through the detection of emission and absorption bands, with the support of laboratory and theoretical investigations, can help identify new species and cast a new light on the DIB conundrum and the organic inventory in space. The detailed spectroscopy of $\mathrm{C}_{60}$ and $\mathrm{C}_{60}^{+}$, which are isolated in the diffuse ISM, can also offer the possibility to study some of the fundamental molecular properties of these species in synergy with laboratory and theoretical investigations.
Acknowledgements. We thank F. Boulanger and E. Falgarone for their thoughtful comments, especially regarding the properties of the studied lines of sight. We thank Alain Omont, for his detailed comments and for identifying a mistake regarding derivation of abundances. We thank the anonymous referee whose comments helped improve the manuscript. This work was supported by the French programme Physique et Chimie du Milieu Interstellaire (PCMI) funded by the Conseil National de la Recherche Scientifique (CNRS) and Centre National d'Études Spatiales (CNES). The research leading to these results has also received funding from the European Research Council under the European Union's Seventh Framework Programme (FP/2007-2013) ERC-2013-SyG, Grant Agreement No. 610256 NANOCOSMOS.

\section{References}

Bakes, E., \& Tielens, A. 1995, in The Diffuse Interstellar Bands, eds. A. G. G. M. Tielens, \& T. P. Snow, Astrophys. Space Sci. Lib., 202, 315

Berné, O., \& Tielens, A. G. G. M. 2012, Proc. Nat. Acad. Sci., 109, 401

Berné, O., Mulas, G., \& Joblin, C. 2013, A\&A, 550, L4

Berné, O., Montillaud, J., \& Joblin, C. 2015, A\&A, 577, A133

Boulanger, F., Bronfman, L., Dame, T. M., \& Thaddeus, P. 1998, A\&A, 332, 273

Cami, J., Bernard-Salas, J., Peeters, E., \& Malek, S. E. 2010, Science, 329, 1180

Cami, J., Bernard-Salas, J., Peeters, E., \& Malek, S. E. 2011, in The Molecular Universe, eds. J. Cernicharo, \& R. Bachiller, IAU Symp., 280, 216

Campbell, E. K., Holz, M., Gerlich, D., \& Maier, J. P. 2015, Nature, 523, 322

Campbell, E. K., Holz, M., Maier, J. P., et al. 2016, ApJ, 822, 17

Castellanos, P., Berné, O., Sheffer, Y., Wolfire, M. G., \& Tielens, A. G. G. M. 2014, ApJ, 794, 83

Cesarsky, C. J., Abergel, A., Agnese, P., et al. 1996, A\&A, 315, L32

Cox, N. L. J., Cami, J., Kaper, L., et al. 2014, A\&A, 569, A117

Dame, T. M., Hartmann, D., \& Thaddeus, P. 2001, ApJ, 547, 792

Diplas, A., \& Savage, B. D. 1994, ApJS, 93, 211

Falgarone, E., Verstraete, L., Pineau Des Forêts, G., \& Hily-Blant, P. 2005, A\&A, 433, 997

Fazio, G. G., Hora, J. L., Allen, L. E., et al. 2004, ApJS, 154, 10

Flagey, N., Boulanger, F., Verstraete, L., et al. 2006, A\&A, 453, 969

Foing, B. H., \& Ehrenfreund, P. 1994, Nature, 369, 296

García-Hernández, D. A., Manchado, A., García-Lario, P., et al. 2010, ApJ, 724, L39

Habing, H. J. 1968, Bull. Astron. Institutes of The Netherlands, 19, 421

Herbig, G. H. 2000, ApJ, 542, 334

Houck, J. R., Roellig, T. L., van Cleve, J., et al. 2004, ApJS, 154, 18

Iglesias-Groth, S., \& Esposito, M. 2013, ApJ, 776, L2

Ingalls, J. G., Bania, T. M., Boulanger, F., et al. 2011, ApJ, 743, 174

Kroto, H. W., \& Jura, M. 1992, A\&A, 263, 275

Kroto, H. W., Heath, J. R., Obrien, S. C., Curl, R. F., \& Smalley, R. E. 1985, Nature, 318, 162

Kupser, P., Steill, J. D., Oomens, J., Meijer, G., \& von Helden, G. 2008, Phys. Chem. Chem. Phys., 10, 6862

McClure-Griffiths, N. M., Pisano, D. J., Calabretta, M. R., et al. 2009, ApJS, 181,398

Menéndez, J., \& Page, J. B. 2000, Vibrational spectroscopy of C60, eds. M. Cardona \& G. Güntherodt (Berlin, Heidelberg: Springer), 27

Mizuno, A., Yamaguchi, R., Tachihara, K., et al. 2001, Proc. Astron. Soc. Japan, 53,1071

Omont, A. 2016, A\&A, 590, A52

Otsuka, M., Kemper, F., Cami, J., Peeters, E., \& Bernard-Salas, J. 2014, MNRAS, 437, 2577

Pilleri, P., Montillaud, J., Berné, O., \& Joblin, C. 2012, A\&A, 542, A69

Roberts, K. R. G., Smith, K. T., \& Sarre, P. J. 2012, MNRAS, 421, 3277

Sassara, A., Zerza, G., Chergui, M., \& Leach, S. 2001, ApJ, 135, 263

Sellgren, K., Werner, M. W., Ingalls, J. G., et al. 2010, ApJ, 722, L54

Smith, J. D. T., Armus, L., Dale, D. A., et al. 2007, PASP, 119, 1133

Sofia, U. J., Lauroesch, J. T., Meyer, D. M., \& Cartledge, S. I. B. 2004, ApJ, 605, 272

Strelnikov, D., Kern, B., \& Kappes, M. M. 2015, A\&A, 584, A55

Tielens, A. G. G. M. 2008, ARA\&A, 46, 289

Walker, G. A. H., Bohlender, D. A., Maier, J. P., \& Campbell, E. K. 2015, ApJ, 812, L8

Werner, M. W., Roellig, T. L., Low, F. J., et al. 2004a, ApJS, 154, 1

Werner, M. W., Uchida, K. I., Sellgren, K., et al. 2004b, ApJS, 154, 309

Wilson, O. C., \& Merrill, P. W. 1937, Contributions from the Mount Wilson Observatory/Carnegie Institution of Washington, 570, 1 


\section{Appendix A: Data analysis}

The data was retrieved from the Spitzer science archive $^{2}$. We used the data at the basic calibrated level (bcd) for both lines of sight. The astronomical observation request (AOR) keys for these observations are, for the four positions of the Scutum line of sight (LoS): 11060992, 12544768, 11061504, 11061760 , from the programme 3513 (PI E. Falgarone), and for the Chamaeleon LoS 28315392, from programme 491 (PI J. Ingalls). The off positions were included in this latter programme, and for the former observations, we used the offs performed the same day for extragalactic sources by another programme (programme 1420; PI L. Armus). The off subtraction, in both cases, was performed at the .bcd level, i.e. before building the 3D spectral cubes. The data reduction was achieved using the CUBISM software (Smith et al. 2007), including the slit loss correction function algorithm ${ }^{3}$. Once the cubes were built, the spectra were obtained by averaging the spectral cubes over all the spatial positions to improve the signal to noise ratio.

The complementary data from NGC 7023 has been discussed extensively in the literature, it was first presented by Werner et al. (2004b). The positions we use to extract the reference spectra are rectangles whose vertices coordinates are given hereafter: for the low resolution spectrum (LL) used in the comparison with the translucent cloud spectrum $(21: 01: 40.579, \quad+68: 10: 42.96 ; \quad 21: 01: 42.343, \quad+68: 10: 54.6$; $21: 01: 40.255,+68: 11: 04.44 ; 21: 01: 38.491,+68: 10: 52.80)$, and for the medium resolution spectrum $(\mathrm{SH})$ used in the comparison with the diffuse ISM spectrum (21:01:24.800, +68:10:11.50; $21: 01: 32.368, \quad+68: 09: 33.95 ; \quad 21: 01: 40.448, \quad+68: 10: 24.60$; 21:01:32.880,+68:11:02.16). The LL spectrum was extracted in the north-west photodissociation region (PDR) of the nebula, where rotational $\mathrm{H}_{2}$ emission is observed to be strong, i.e. at the surface of the molecular cloud. The high resolution spectrum was obtained by averaging the spectral cube over a region that covers the north-west PDR and the cavity of atomic gas situated close to the illuminating star HD 200775.

\section{Appendix B: Derivation of neutral $\mathrm{C}_{60}$ abundances from the infrared emission}

The derivation of the $\mathrm{C}_{60}$ abundance performed here relies on the assumption that the energy absorbed by $\mathrm{C}_{60}$ in the UV is completely re-radiated in the IR bands. For moderate internal energies $(\sim 5-15 \mathrm{eV})$, such as those of $\mathrm{C}_{60}$ molecules excited by Far-UV photons in the ISM, the main relaxation channel is indeed IR emission (see Fig. 2 in Berné et al. 2015) and this assumption is valid. The total IR intensity in $\mathrm{W} \mathrm{m}^{-2} \mathrm{sr}^{-1}$ emitted by $\mathrm{C}_{60}$ molecules is hence,

$I_{\mathrm{tot}}=N\left(\mathrm{C}_{60}\right) \times \sigma_{\mathrm{UV}} \times G_{0} \times 1.2 \times 10^{-7}$,

where $N\left(\mathrm{C}_{60}\right)$ is the column density of $\mathrm{C}_{60}, \sigma_{\mathrm{UV}}$ is the $\mathrm{UV}$ absorption cross section of $\mathrm{C}_{60}$ which we take to be $4.2 \times 10^{-16} \mathrm{~cm}^{2}$ following Berné \& Tielens (2012), and $G_{0}$ is the radiation field (see Table 1). Unfortunately, in our observations, the bands at shorter wavelengths $(7.0$ and $8.5 \mu \mathrm{m})$ are not detected, and the $17.4 \mu \mathrm{m}$ band is contaminated by PAH emission, hence $I_{\text {tot }}$ cannot be derived directly. Instead, it can be estimated using the $18.9 \mu \mathrm{m}$ band intensity $\left(I_{18.9}\right)$, assuming that $I_{\text {tot }}=2.3 \times I_{18.9}$, which is what is observed in NGC 7023 (Berné et al. 2013). This

\footnotetext{
2 http://sha.ipac.caltech.edu/applications/Spitzer/ SHA/

3 http://tir.astro.utoledo. edu/jdsmith/code/cubism. php
}

assumption is valid considering that the band ratios of the IR bands of $\mathrm{C}_{60}$ depend on the average energy of absorbed UV photons that are expected to be comparable in NGC 7023 and in the diffuse ISM, since both environments are dominated by the UV fields of young massive stars. Hence, one can solve Eq. (B.1) for $N\left(C_{60}\right)$, and derive the abundance of $\mathrm{C}_{60}$ relative to carbon as follows:

$f_{\mathrm{C}}^{\mathrm{C}_{60}}=\frac{N\left(\mathrm{C}_{60}\right) \times 60}{N(\mathrm{H}) \times[\mathrm{C}]}$

adopting a carbon-to-hydrogen fraction measured in the diffuse ISM, i.e. [C] $=1.6 \times 10^{-4}$ (Sofia et al. 2004), and the values for $N(\mathrm{H})$ in Table 1 . For the specific cases considered here, we derive $I_{18.9}=2.5 \times 10^{-10}$ and $I_{18.9}=1.2 \times$ $10^{-8} \mathrm{~W} \mathrm{~m}^{-2} \mathrm{sr}^{-1}$ for the Chamaeleon and Scutum LoS respectively. Using Eqs. (B.1), (B.2) and the values in Table 1 (including uncertainties on the hydrogen column density and radiation field) yields abundances of $\mathrm{C}_{60}$ of $0.3-2.4 \times 10^{-3}$ and $0.4-3.6 \times 10^{-3}$ of the gas-phase carbon locked in $\mathrm{C}_{60}$, for the Chamaeleon and Scutum LoS, respectively.

\section{Appendix $\mathrm{C}: \mathrm{C}_{60}^{+}$abundances in the diffuse interstellar medium}

The fraction of carbon locked in $\mathrm{C}_{60}^{+}$relative to gas-phase carbon is given by

$f_{\mathrm{C}}^{\mathrm{C}_{60}^{+}}=\frac{N\left(\mathrm{C}_{60}^{+}\right) \times 60}{N(\mathrm{H}) \times[\mathrm{C}]}$

adopting a carbon-to-hydrogen fraction measured in the diffuse ISM, $[\mathrm{C}]=1.6 \times 10^{-4}$. Values of the column density of $\mathrm{C}_{60}^{+}$, $N\left(\mathrm{C}_{60}^{+}\right)$, were derived in previous works along several diffuse ISM LoS along which the two DIBs at 9577 and $9632 \AA$ were observed. In Table C.1, we report the values for three well-studied LoS and derived abundances for $\mathrm{C}_{60}^{+}$using the equation above. The derived values are of the order of a few $10^{-3}$ of the gasphase carbon. Omont (2016) finds an average value of the fraction of carbon contained in $\mathrm{C}_{60}^{+}$(with respect to the total carbon abundance, including dust) $X\left(\mathrm{C}_{60}^{+}\right)=4 \pm 2 \times 10^{-4}$, equivalent to $f_{\mathrm{C}}^{\mathrm{C}_{60}^{+}} \sim 1 \times 10^{-3}$ of the gas phase carbon, in agreement with our results.

\section{Appendix $D: C_{60}^{+}$infrared emission upper limit}

It is possible to derive a limit for the abundance of $\mathrm{C}_{60}^{+}$based on the infrared spectra of the Chamaeleon and Scutum LoS using the same approach as for $\mathrm{C}_{60}$ (see appendix $\mathrm{B}$ ). The $7.1 \mu \mathrm{m}$ band of $\mathrm{C}_{60}^{+}$is the easiest to detect (Berné et al. 2013), however it is absent in both lines of sight (Fig. 1). Based on these non-detections, we can place an upper limit on $I_{7.1}$, which we can convert into an $I_{\text {tot }}$ for $\mathrm{C}_{60}^{+}$using the band ratio observed in NGC 7023 (Berné et al. 2013). We derive an upper limit for the $\mathrm{C}_{60}^{+}$column density, which we convert into an abundance using Eq. (B.2). Using the equation of energy budget (Eq. (B.1)) and assuming a similar value of the UV absorption cross section for $\mathrm{C}_{60}$ and $\mathrm{C}_{60}^{+}$, we can derive an upper limit for the $\mathrm{C}_{60}^{+}$column density, which we convert into an abundance using Eq. (C.1). This yields an upper limit for the $\mathrm{C}_{60}^{+}$abundance of $3 \times 10^{-3}$ of the elemental carbon abundance in the Chamaeleon line of sight and $\sim 1.8 \times 10^{-3}$ of the carbon for the Scutum line of sight. Both limits are above the measurements of $\mathrm{C}_{60}^{+}$abundance resulting from the detection of the electronic transitions (Table C.1). 
Table C.1. $\mathrm{C}_{60}^{+}$column densities and abundances (fraction of gas-phase carbon) for several well-studied diffuse ISM lines of sight.

\begin{tabular}{lllll}
\hline \hline Line of sight & $N\left(\mathrm{C}_{60}^{+}\right)^{*}$ & Ref. for 9577 DIB EW & $N(\mathrm{H})^{* *}$ & $\mathrm{C}_{60}^{+}$abundance $f_{\mathrm{C}}$ \\
\hline HD169454 & $0.9 \times 10^{13}$ & Walker et al. (2015) & $3 \times 10^{21}$ & $1.1 \times 10^{-3}$ \\
HD183143 & $2 \times 10^{13}$ & Walker et al. (2015) & $7.4 \times 10^{21}$ & $1.0 \times 10^{-3}$ \\
4U1907+09 & $2.6 \times 10^{13}$ & Cox et al. (2014) & $1.7 \times 10^{22}$ & $6.0 \times 10^{-3}$ \\
\hline
\end{tabular}

Notes. ${ }^{(*)}$ Column density computed from the measured equivalent width via the measured cross sections given by Campbell et al. (2016). ${ }^{(* *)}$ Hydrogen column density, $N(\mathrm{H})=N\left(\mathrm{HI}+2 N\left(\mathrm{H}_{2}\right)\right.$, from direct measurements (HD 169454: Diplas \& Savage 1994) or visual extinction, $A_{\mathrm{V}}$ (other sightlines; see text for details).

Table E.1. Wavelength, oscillator strength, and FWHM of the considered $\mathrm{C}_{60}$ electronic bands (all values from Sassara et al. 2001).

\begin{tabular}{lll}
\hline \hline$\lambda(\AA)$ & $f$ & $F W H M(\AA)$ \\
\hline 3980 & 0.007 & 5.5 \\
4024 & 0.005 & 4.0 \\
\hline
\end{tabular}

\section{Appendix E: Prediction of neutral $\mathrm{C}_{60}$ DIB strength}

The detection of the IR emission of neutral $\mathrm{C}_{60}$ in the diffuse ISM with abundances comparable to $\mathrm{C}_{60}^{+}$suggests that this molecule could be a DIB carrier. Using the abundances derived in this paper and relevant data for the positions, strength, and widths of the electronic transitions of $\mathrm{C}_{60}$ (Sassara et al. 2001, Table E1), it is possible to compute the predicted depth of the electronic transitions of neutral $\mathrm{C}_{60}$. Truly accurate positions, oscillator strengths, and band widths have not yet been determined for the electronic absorption bands of neutral $\mathrm{C}_{60}$ in the gas phase at low temperatures, which could therefore be shifted by several $\AA$ with respect to these recommended values. We use the following relation between column density, $N$, and equivalent width, EW in $\AA$ (Wilson \& Merrill 1937):

$$
E W=\frac{N f \lambda^{2}}{1.13 \times 10^{20}},
$$

where $\lambda$ is the band position (in $\AA$ ) and $f$ its oscillator strength (cf. Table E.1).

For a Gaussian profile the central depth, $\tau_{0}$, of the line centre relative to the local continuum, is given as

$$
\tau_{0}=\frac{E W}{1.571 \times F W H M} .
$$

For the diffuse Scutum LoS, the column density derived from infrared emission is $N\left(\mathrm{C}_{60}\right)=5.6 \times 10^{14} \mathrm{~cm}^{-2}$ for a sightline with total visual extinction, $A_{\mathrm{V}}$, of 30.5 magnitudes. For the Chamaeleon LoS, with $A_{\mathrm{V}}=1-2$ mag, we have $N\left(\mathrm{C}_{60}\right)=$ $1.7 \times 10^{13} \mathrm{~cm}^{-2}$. We can thus infer $N\left(\mathrm{C}_{60}\right) \sim 1 \times 10^{13} \mathrm{~cm}^{-2} / A_{\mathrm{V}}$ from both sightlines. Using Eqs. (E.1) and (E.2), this yields central depths of $\sim 0.1 \%$ per unit visual extinction. These low values are inherent to the rather low oscillator strengths of the $\mathrm{C}_{60}$ electronic transitions and suggest that $\mathrm{C}_{60}$ is unlikely to be responsible for strong DIBs, which can be several percent of the continuum (Herbig 2000). However, weak DIBs, which can be detected thanks to highly sensitive spectroscopy, could well be attributed to $\mathrm{C}_{60}$ with the help of new gas-phase laboratory spectroscopy to provide accurate positions, width, and strengths for the electronic transitions. For instance, on the Scutum LoS, the predicted equivalent width for the $\mathrm{C}_{60}$ DIBs with the number quoted above is of the order of $200 \mathrm{~m} \AA$ provided one can find a proper background star.

\section{Appendix F: Possible contribution of $\mathrm{C}_{60}^{+}$ to the $18.9 \mu \mathrm{m}$ band}

$\mathrm{C}_{60}^{+}$is known to have an infrared vibrational band at a wavelength close to the strongest band of $\mathrm{C}_{60}$, i.e. at $\sim 18.9 \mu \mathrm{m}$ (see theoretical calculations in Berné et al. 2013, and experimental data in Strelnikov et al. 2015). Based on the upper limits for the $7.1 \mu \mathrm{m}$ band (Fig. 1), it is possible to derive an estimate of the maximum contribution of $\mathrm{C}_{60}^{+}$to the $18.9 \mu \mathrm{m}$ band (Fig. 2). A proper analysis would require a detailed emission model, including a description of the UV field, absorption, excitation, and cooling of the molecules. Here we consider a simplified model. From the observed ratio between the 7.0 and $18.9 \mu \mathrm{m}$ bands of $\mathrm{C}_{60}$ in NGC 7023 (Sellgren et al. 2010), we can derive a mean colour temperature $T_{\mathrm{C}}$ such that

$\frac{I_{7.0}}{I_{18.9}}=0.83=\frac{\sigma_{\mathrm{C}_{60}}(7.0) \times B\left(7.0, T_{\mathrm{C}}\right)}{\sigma_{\mathrm{C}_{60}}(18.9) \times B\left(18.9, T_{\mathrm{C}}\right)}$,

where $B(\lambda)$ is the Planck function and $\sigma$ is the infrared cross section of the molecule. Applying a similar reasoning for $\mathrm{C}_{60}^{+}$and assuming the same colour temperature for both $\mathrm{C}_{60}$ and $\mathrm{C}_{60}^{+}$, the ratio between the 7.1 and $18.9 \mu \mathrm{m}$ features reads

$$
\frac{I_{7.1}}{I_{18.9}}=\frac{\sigma_{\mathrm{C}_{60}^{+}}(7.1) \times B\left(7.1, T_{\mathrm{C}}\right)}{\sigma_{\mathrm{C}_{60}^{+}} 18.9 \times B\left(18.9, T_{\mathrm{C}}\right)}=\frac{\sigma_{\mathrm{C}_{60}^{+}}(7.1) \times \sigma_{\mathrm{C}_{60}}(18.9)}{\sigma_{\mathrm{C}_{60}^{+}}(18.9) \times \sigma_{\mathrm{C}_{60}}(7.0)} \times 0.83 .
$$

Using the IR cross sections for $\mathrm{C}_{60}$ and $\mathrm{C}_{60}^{+}$of Menéndez \& Page (2000) and Strelnikov et al. (2015), respectively, yields a theoretical value of $I_{7.1} / I_{18.9} \sim 9$. The upper limits for the $7.1 \mu \mathrm{m}$ band in the Chamaeleon and Scutum LoS are $5.9 \times 10^{-10}$ and $1.2 \times 10^{-8} \mathrm{~W} \mathrm{~m}^{-2} \mathrm{sr}^{-1}$, respectively. This results in a maximum contribution of $\mathrm{C}_{60}^{+}$to the $18.9 \mu \mathrm{m}$ band of $6.6 \times 10^{-11}$ and $1.3 \times 10^{-9} \mathrm{~W} \mathrm{~m}^{-2} \mathrm{sr}^{-1}$, respectively. This is to be compared to the observed emissions of the $18.9 \mu \mathrm{m}$ band of $2.5 \times 10^{-10}$ and $1.2 \times 10^{-8} \mathrm{~W} \mathrm{~m}^{-2} \mathrm{sr}^{-1}$. Hence, the contribution of $\mathrm{C}_{60}^{+}$to the $18.9 \mu \mathrm{m}$ band is at most $\sim 25$ and $10 \%$ in the Chamaeleon and Scutum LoS, respectively. In any case, the major contributor to the $18.9 \mu \mathrm{m}$ band is $\mathrm{C}_{60}$. 